\title{
A Secure and Semi-Blind Technique of Embedding Color Watermark in RGB Image Using Curvelet Domain
}

\author{
Ranjeeta \\ CSE Department, Thapar University, Patiala-147001, Punjab, India \\ E-mail: er.ranjeetaved@gmail.com \\ Dr. Sanjay Sharma* and Dr. L. R. Raheja ${ }^{+}$ \\ *ECE Department, Thapar University, Patiala-147001, Punjab, India \\ ${ }^{+}$Navel Department, IIT Kharagpur, Kharagpur, West Bengal 721302, India \\ E-mail: sanjay.sharma@thapar.edu,lrr.2004@yahoo.com
}

\begin{abstract}
A semi-blind and secure watermarking technique for the color image using curvelet domain has been proposed. To make the algorithm secure a Bijection mapping function has been used. The watermark also separated into color planes and each color plane into a bit planes. The most significant bit (MSB) planes of each color used as the embedding information and remaining bit planes are used as a key at the time of extraction. The MSB planes of each color of watermark image embedded into the curvelet coefficients of the blue color plane of the processed cover image. For embedding the MSB bit planes of watermark image in the cover image each curvelet coefficient of blue planes of the processed cover image has been compared with the value of its 8 connected coefficients (neighbors). The results of the watermarking scheme have been analyzed by different quality assessment metric such as PSNR, Correlation Coefficient (CC) and Mean Structure Similarity Index Measure (MSSIM). The experimental results show that the proposed technique gives the good invisibility of watermark, the quality of extracting watermark and robustness against different attacks.
\end{abstract}

Index Terms-Digital watermarking, Curvelet transform, Bit-plane, MSSIM.

\section{INTRODUCTION}

To protect the interest of the author digital watermarking is used. In digital watermarking embed the watermark in the original work such that it preserves the quality of watermarked data. The watermark can later be extracted for the purpose of author's identification. There are two domains for embedding the watermark one is spatial domain and other is transformed domain. In the first method of embedding, the watermark is directly embedded by changing the original pixel intensities of the cover image. A possible disadvantage of spatial techniques is that they do not give robustness of the watermark [1-2]. Transformations involved in watermarking are common, which include Fourier transform [3], Discrete Cosine Transform (DCT) [4-6], Digital Wavelet Transform (DCT) [7-10], Ridgelet Transform (RT) [11] and much more.

Cox et al. [2] renowned that watermark to be robust against the image processing operations, it must be located in perceptually important regions of the image. Watermarking is based on samples and embedded into largest DCT coefficients. Gunjalet. Al. [8] proposed a robust and highly secured DWT-SVD based color image watermarking in all Y, U, V color spaces. Peining Tao [9] proposed watermarking using wavelet transformation which provides multi-resolution representation of the image.

Curvelet transform [12] for has properties such as it provide sparse representations of objects along a curve and it is anisotropic with strong direction. The curvelet transform is a multi-scale pyramid with many directions and positions of each fine scale and has needle-shaped elements at the fine scales. Thai Hien al. [14] embedded the watermark in the curvelet transform which contains as much edge information as possible. It has good invisibility but poor robustness. Shi et al. [15] proposed a semi-fragile watermarking algorithm by embedding the watermark in the supreme model of curvelet coefficient. This algorithm recollects good robustness against a compression operation.Among these techniques, those require the original cover image, key and bits of watermark for watermark extraction are called non-blind watermarking techniques [4]. Robustness of non-blind technique is good. But it is not suitable for watermark detection in DVD player because the original data is not available. Those techniques need the secret key but not require original cover image at the time of extraction is called a blind watermarking technique. But the blind watermarking compares usually less robust compare with non-blind watermarking. Those requiring the secret key and the watermark bit sequence are called semi-blind watermarking technique. Semi-blind watermarking gives yield good robust against attacks and suitable for most of the application. 
Literature also gives some semi-blind technique Lin et al. [16] proposed a semi-blind watermarking scheme using the discrete Fourier transform. Solachidis al [17] uses circular symmetric in DFT domain. Stankovic al. [18] embed the watermark by mean of a $2 \mathrm{D}$ radon - Wigner distribution.. Some of the authors take the colored image as the cover image, to best of author's knowledge no one yet tried to embed the color watermark in the color cover image using curvelet transform. Here we proposed secure and semi-blind watermarking technique. To make secure watermark a key that is a Bijection function is being used to split a cover image into blocks. By this key, the cover image is disturbed. A bit plane method is also used to separate the watermark colors and bits planes of each color plane. The Most Significant Bit (MSB) planes of each color planes are used as embedding information. Embedding is done by analyzing the coefficient values of neighbor's coefficients in the blue color plane of curvelet domain.

\section{Curvelet Transform}

This block ridgelet-based transform is called as curvelet transform. It was first proposed by Candes and Donoho [12] . Actually, the ridgelet transform is fundamental for the curvelet transform. The ridgelet transform is optimal at representing straight-line singularities. Unfortunately, worldwide straight-line singularities are rarely used in many applications. To analyze local line or curve singularities, a natural idea is to consider a partition of the image, and then to apply the ridgelet transform to the obtained sub-images. So the block based ridgelet transform is named as curvelet transform[19-22].Let $\mu$ be the triple $(j, l, k)$ in the function plane. Where $j=0,1 \ldots$ is a scale parameter, $l=0,1,2 \ldots . \quad$ an orientation parameter and $k=\left(k_{1,} k_{2},\right) ; k_{1}, k_{2}, \in Z$ is the translation parameter pairs. A curvelet coeffiecits is simply the inner product between an $f \in L^{2}\left(R^{2}\right) \quad$ element $f \in L^{2}\left(R^{2}\right)$ and curvelet
$\quad 1 \leq i \leq M, 1 \leq j \leq N$
$\phi_{m}$ given by equation (1).

$$
C_{\mu}=f, \phi_{\mu}=\int f(x) \phi_{\mu} d x=\frac{1}{(2 \pi)^{2}} \int \hat{f}(w) U_{j}\left(R_{\theta l} w\right) e^{i x_{k}^{J}, w}
$$

Where $R_{\theta}$ is the rotation by $\theta$ radian, $J=(j, l)$ is the index of wedge for all $k$ with it and $U_{j}$ is a polar wedge window of radial dilation and angular transformation[17]. Define coarse scale curvelet as in equations (2) and (3).

$$
\phi_{j, 0, k}=\phi_{j, 0}\left(x-2^{-j, 0, k}\right)
$$

$$
\hat{\phi_{j, 0}}(w)=2^{-j 0} A_{0}\left(2^{-j, 0}|w|\right)
$$

Similar to other multi-scale pyramids, curvelet transform images into several frequency scales. An important factor in curvelet domain is not the "Approximation Rate". If having an object in the domain $[0,1] \times[0,1]$,how fast can approximate it using the certain system of function? In comparing each transforms method and illustrate that the curvelet transform is the best.

$$
\begin{gathered}
f-\tilde{f}_{m_{2}}^{2}=O\left(m^{-\frac{1}{2}}\right) \\
f-\tilde{f}_{m_{2}}^{2}=O\left(m^{-1}\right) \\
f-\tilde{f}_{m_{2}}^{2}=O\left(m^{-2} \log ^{3} m\right)=O\left(m^{-2}\right)
\end{gathered}
$$

Equation (4), equation (5) and equation (6) gives the approximate rate of Fourier Transform, wavelet transform, and curvelet transform respectively. Conceptually the curvelet transform is a multi-scale pyramid with many directions and positions at each fine scale and needleshaped elements at fine scales [20-22]. Based on the curvelet transform, it is possible to embed watermarks onto more significant components and spread the amendments to more space locations. The implementation of digital curvlelet transform there are two ways.

1) Curvelet via USFFT (Unequally Spaced Fast Fourier Transform)

2) Curvelet via wrapping.

Both the ways are linear and take as input a cartesian array $f\left[t_{1}, t_{2}\right], 0 \leq t_{1}, t_{2}<n$ and give the discrete coefficients. Uses of digital coronization are same in both, but they differ in choice of spatial grid to translate curvelet at each scale and angle. The time complexities of both implementations are $\mathrm{O}\left(n^{2} \log n\right)$ for $n * n$ Cartesian array. In this paper we use FDCT via USFFT. It is not possible to delve in to the USFFT within the scope of this paper; interested reader may consult the work of Candes \& Doncho [20-22].

\section{A Properties of curvelet beneficial}

Fourier transform not well define the discontinuities. It requires a large number of terms to reconstruct a discontinuity. Wavelet transform are localized and multiscale that solve singularity problem. It good for 1-D, but due to the limited orientation it fail to represent higher dimensional singularity. In wavelet there are fixed number of directional elements that the reason it do not 
exhibit highly anisotropic elements. Images do not always exhibit isotropic scaling and these limitations must be overcome. Curvelet has been developed specially to represent objects with curve punctuated smoothness", as said above that wavelet representation point singularity in 1D, 2D may efficiently but fail to represent curve singularity. Less curvelet coefficients are required to represent edges because of its elongated needle shape. Curvelet is based on anisotropic scaling principle.

To summarize, wavelet transform suffer from following limitations:-

1) Edge representation

2) Crude directional elements independent to scale

3) No highly anisotropic elements

Curvelet transform is an improvement on wavelet and overcomes this limitation of wavelet. Digital curvelet transform is a wavelet transform applied to coronae of the image which thus incorporates the angel of the coronae in the transform as shown in Fig. 1. The 2D image is taken as signal in time domain. The image is divided into wedges (coronae) by drawing concentric squares $\&$ radial lines as shown in Fig.1. This forms a grid which is a replacement of polar grid used for continuous curvelet transform. Next, 2D FFT is applied to the image treated as time domain signal. Now each corona which is a cartesian array of frequencies obtained by FFT is subjected to inverse 1D FFT so as to get time domain values. Subsequently; a wavelet transform is applied to each corona at different scales and translations along the radial lines but for fixed angle of the coronae. This procedure is repeated for various angles to exhaust the entire coronae. The frequencies obtained in this way at different scales, translations and angles are the frequencies of curvelet transform.

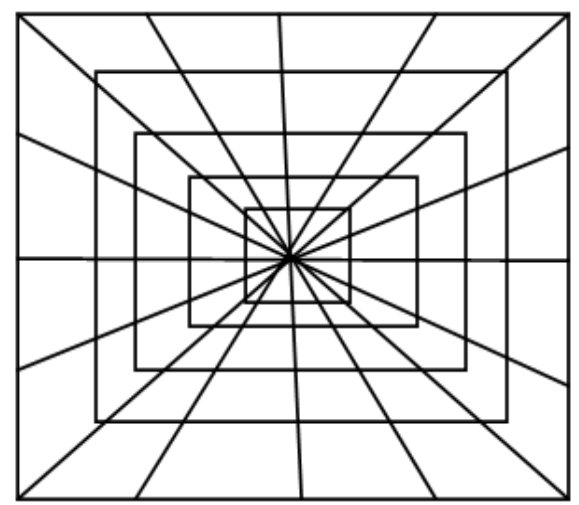

Fig.1. Cartesian array on input

The procedure described above is depicted in the flow chart below for better understanding [15].

The Fig 4-5 give an image with curvelet grid and the corresponding curvelet transform coefficients respectively. The low frequency coefficients are stored at the centre of the display. The cartesian concentric coronae show the coefficients at different scale. The outer coronae correspond to higher frequencies

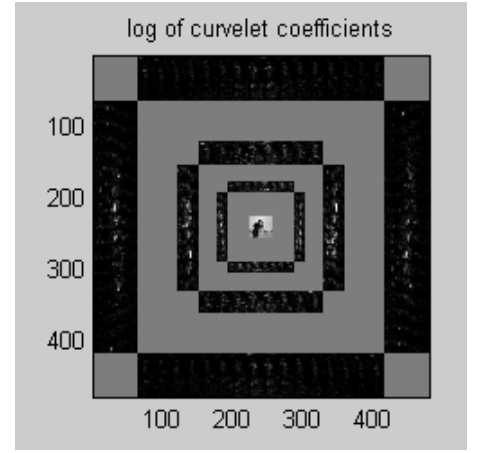

Fig.2. Curvelet Transform

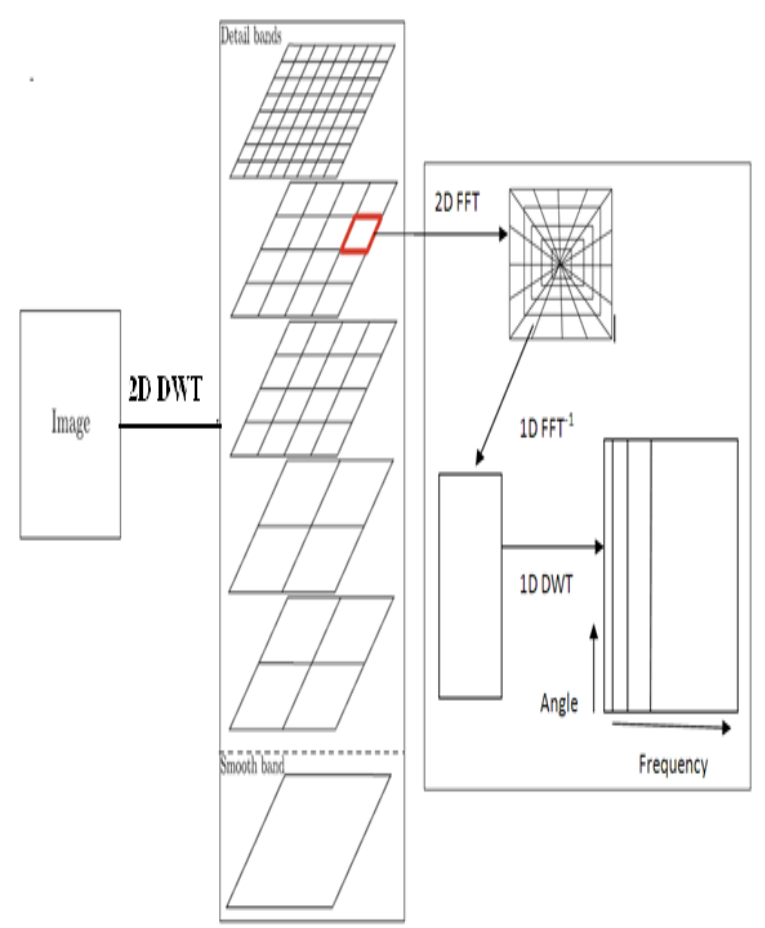

Fig.3. Discrete Curvelet Transform flowchart

\section{Proposed Watermark TechniQue}

A secure watermarking scheme has been proposed in this section. To make watermark secure a Bijection mapping function is used to split the image into $8 * 8$ blocks.

\section{A. Cover Image Preprocessing}

A secure watermarking scheme has been proposed in this section. To make watermark secure a bijection mapping function is used to split the image into $8 * 8$ blocks.

1. Split the cover image I into RGB color planes. Ired ,Igreen, Iblue represent the red, green and blue color plane of the original cover image respectively.

2. Split the each color plane of the cover image into $8 * 8$ non-overlapping blocks. Give a unique serial number to each block. Denoted by Blockseq 
3. for each color plane

- Let $\mathrm{L}$ is the largest serial number, and 1 is the mid of series

- For each block, If $(\bmod (B$ lockseq, 2$)=0)$ then newseq $=\mathrm{L}$ and $\mathrm{L}=\mathrm{L}-1$; Else newseq $=1$ and $l=l-1$;

4. Combine the blocks with new sequences. This generates the new modified cover image.

\section{B. Watermark image preprocessing}

Watermark image is also colored so firstly separates their color planes $\mathrm{Br}, \mathrm{Bg}$ and $\mathrm{Bb}$ red plane, green plane and the blue plane of the watermarked image respectively. Now obtain bit planes of each color plane. $\left\{B r_{0}, B r_{1} \ldots B r_{7}\right\},\left\{B g_{0}, B g_{1} \ldots B g_{7}\right\} \quad$ and $\left\{B b_{0}, B b_{1} \ldots B b_{7}\right\}$ are the set of bit planes of red, green and blue color planes respectively. $\mathrm{Br}_{0} \cdot \mathrm{Bg}_{0}$ and $\mathrm{Bb}_{0}$ are the least significant bit of red, green and blue color. $\mathrm{Br}_{7}$, $\mathrm{Bg}_{7}$ and $\mathrm{Bb}_{7}$ are most significant bit plane of red, green and blue color used as the watermark (to be embedded) and remaining other $\left\{B r_{0}, B r_{1} \ldots B r_{6}\right\},\left\{B g_{0}, B g_{1} \ldots B g_{6}\right\}$ and $\left\{B b_{0}, B b_{1} \ldots B b_{6}\right\}$ are using a key at the time of extraction

\section{Embedding Algorithm}

1. Apply selected scale and perform curvelet transform on the blue color plane (Wb) of watermarked image. Stored the curvelet coefficient let Ex

2. Apply key i.e selected scale and orientation where watermark has been embedded. Let selected scale 1 and orientation $k_{1}, k_{2}, k_{3}$ for red, green and blue bit-planes.

3. Let D1, D2 and D3 are the selected scale and orientation for embedding the red, green and blue watermark bit plane.

4. For each selected scale and orientation

5. Compare the value of coefficient with its 8 neighbor coefficient. And lets $\mathrm{Nr}, \mathrm{Ng}$ and $\mathrm{Nb}$ denotes the total number of neighbors having values less than coefficient of red, green and blue planes respectively

6. For each color plane use equations (7), (8) and (9)
a. If( $\quad\left(N r>=4 \& \& B r_{7}=1 \quad\right) \quad \& \&$$$
\left.\mathrm{Nr}<4 \& \& \mathrm{Br}_{7}=0\right) \text { )than } \mathrm{Ur}=1
$$$$
U r=-1
$$$$
\text { b. If } \quad\left(\left(\mathrm{Ng}>=4 \& \& B g_{7}==1\right)\right.
$$$$
\left.\left(\mathrm{Ng}<4 \& \& \mathrm{Bg}_{7}=0\right)\right) \text { than } U g=1
$$$$
U g=-1
$$$$
\text { c. If } \quad\left(\left(N b>=4 \& \& B b_{7}=1\right)\right.
$$$$
\left(\mathrm{Nb}<4 \& \& B b_{7}==0\right)
$$$$
\text { Than }
$$

$$
U b=-1
$$

7. For each coefficient of color planes (Embedding),

8. $O r=D 1+\alpha^{*} U r \quad, \quad O g=D 2+\alpha * U g$, $O b=D 3+\alpha * U b$,

9. Where $\alpha$ is the embedding strength parameter.

10. Perform inverse transform on resulted blue plane to convert frequency domain color plane into the time domain.

11. Combine the entire three color plane and make it RGB image

12. Perform step A to resulted image in reverse way to get the watermarked image

\section{Extraction Algorithm}

A semi-blind method of extraction has been discussed in which we required a little part of information regarding watermark but no need of the cover image.

1. Read watermarked image $\mathrm{W}$,

2. Apply image preprocessing describes in B on the watermarked image.

3. Let E1, E2 and E3 are the metros of selected scale and orientation for embedding the red, green and blue watermark bit plane.

4. For each selected scale and orientation

a. Calculate the value of $\mathrm{E}$ for each coefficient with 8 neighbors. $\mathrm{Er}, \mathrm{Eg}$ and $\mathrm{Eb}$ denote the total number of neighbors having values less than $C(i, j)$ of red, green and blue planes respectively.

b. For each color plane use equations (11),(12) and (13).

$$
\begin{aligned}
& \text { a) If } \quad((E r>=4 \& \& U r=1) \quad \& \& \\
& (E r<4 \& \& U r=-1)) \text { than } E x r=1 \text { else } \\
& \text { Exr }=0 \\
& \text { b) If } \quad((E g>=4 \& \& U g==1) \quad \& \& \\
& (E g<4 \& \& U g=-1)) \text { Than } E x g=1 \text { else } \\
& \operatorname{Exg}=0 \\
& E x b=0
\end{aligned}
$$

5. To extract color watermark from the computed information use equations (14), (15) and (16).

$$
\begin{aligned}
& \text { a. } \quad \text { Extr }=\sum_{i=0}^{7} \operatorname{Ext}^{*} 2^{7}+B r_{i} \\
& \text { b. } \quad \operatorname{Extg}=\sum_{i=0}^{7} \operatorname{Exg}^{*} 2^{7}+B g_{i}
\end{aligned}
$$


c. $\quad E x t b=\sum_{i=0}^{7} E x b^{*} 2^{7}+B b_{i}$

6. Combine these color planes and get the RGB extracted the watermark.

\section{Performance Evaluation Metric}

Embedding the watermark in the original image may degrade the visual quality of the image as well as the extraction procedure may not be extracting the exactly identical watermark with the original watermark. So a standard is required to determine the degree of similarity between the original image and watermarked image. The subjective approach is most effective for the meaningful logotype watermark. In this paper, except for the subjective approach, the Peak Signal to Noise Ratio (PSNR) shown in equation (17), Normalized Correlation (NC) shown in equation (18)and Mean Structure and Similarity Index Measure (MSSIM, are used to evaluate the degree of similarity between the original watermark $\mathrm{W}$ and the extracted watermark $w^{*}$.

$$
\begin{gathered}
P S N R=10 \log \log \left(\frac{255^{2}}{\frac{1}{M * N} \sum_{i, j}(X(i, j)-\hat{X}(i, j)}\right) \\
N C=\frac{\sum_{i, j}(X(i, j) * \hat{X}(i, j)}{\sum_{i, j} X(i, j)^{2}}
\end{gathered}
$$

Where $1 \leq i \leq M, 1 \leq j \leq N$, the $\mathrm{M}$ and $\mathrm{N}$ are rows and column of the image.

Although PSNR is convenient to calculate and also has clear physical meaning but still it does not correlate strongly enough with the visual quality of the image for most applications [23]. The scope of perceptual quality is widened in the Mean Structure Similarity Index Measure (MSSIM) [24-25].MSSIM is the combination of three perceptual properties namely Luminance, Contrast and structure at every point of the two images being compared. For the purpose of calculation of MSSIM the image to be compared are broken into a number of windows of $\mathrm{B} * \mathrm{~B}$ size, further the two images must be of the same size [24]. If $x$ and $y$ are two images, $M$ is the no. of $\mathrm{B} * \mathrm{~B}$ windows and $\mathrm{N}$ is the number of pixels in each window then SSIM is defined in equation (19).

$$
\operatorname{SSIM}=\frac{\left(2 \mu_{x} \mu_{y}+c_{1}\right)\left(2 \sigma_{x y}+c_{2}\right)}{\left(\mu_{x}^{2}+\mu_{y}^{2}+c_{1}\right)\left(\sigma_{x}^{2}+\sigma_{y}^{2}+c_{2}\right)}
$$

Where $\mu_{x}=\frac{1}{N} \sum^{i=1 N} x_{i}, \mu_{y}=\frac{1}{N} \sum^{i=1 N} y_{i}$

$$
\sigma_{x}=\sqrt{\left(\frac{1}{N} \sum_{i}^{N}\left(x_{i}-\mu_{x}\right)^{2}\right)}, \sigma_{y}=\sqrt{\left(\frac{1}{N} \sum_{i}^{N}\left(y_{i}-\mu_{x}\right)^{2}\right)}
$$

$$
c_{1}=\left(k_{1} L\right)^{2}, c_{2}=\left(k_{2} L\right)^{2} \text { In paper [26] } k_{1}=0.01 \text { ， }
$$

$k_{2}=0.03$ has been proposed

$L=$ Dynamic range of the pixel values (255 for 8 bit gray scale image).

MSSIM (Mean SSIM) is defined in equation (20).

$$
\operatorname{MSSIM}=\frac{1}{M} \sum^{j=1 M} \operatorname{SSIM}\left(x_{j}, y_{j}\right)
$$

\section{EXPERIMENTAL RESULTS}

To test the algorithm colored "lena.jpg" image used a cover image and a colored "thaper.jpg" image used a watermark image. Fig. 4 and Fig.5 show the "lena.jpg" and "thaper.jpg" image respectively. In the algorithm firstly the cover image is processed using some bijectional method. Fig. 6 shows the processed cover image. Fig. 7 shows the resulting watermarked image. The algorithm has been tested against all the requirement parameter such as invisibility, effectiveness and robustness.

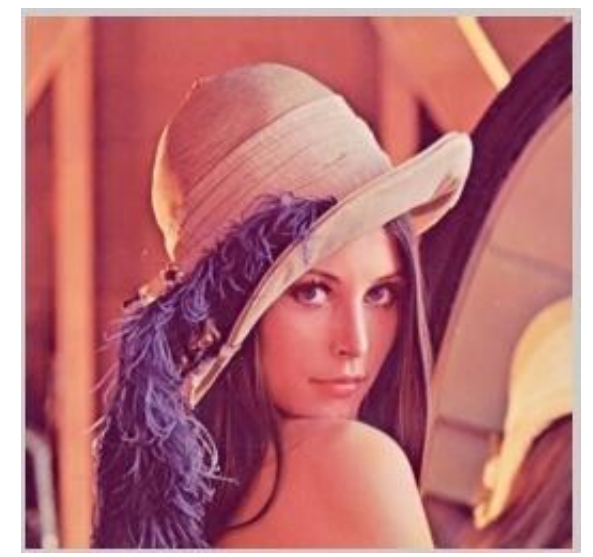

Fig.4. lena.jpg

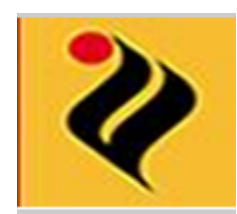

Fig.5. thapar.jpg watermark 


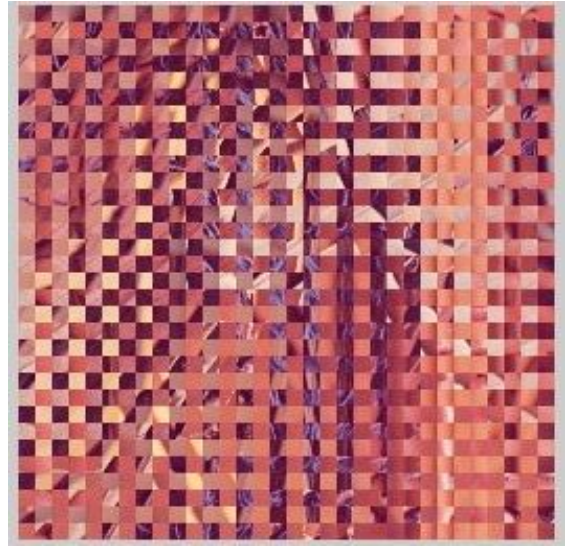

Fig.6. Processed cover image

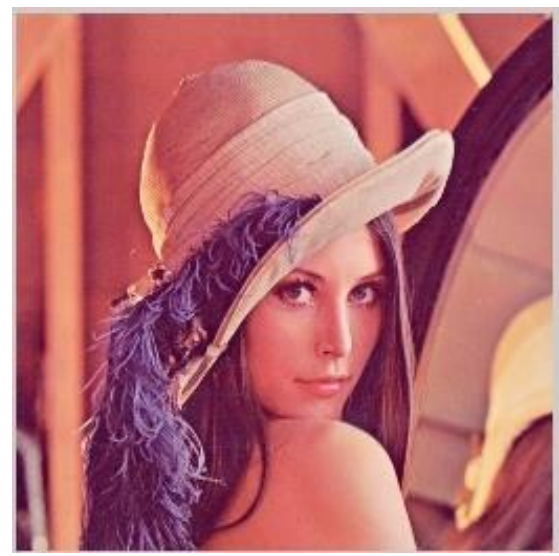

Fig.7. Watermarked image

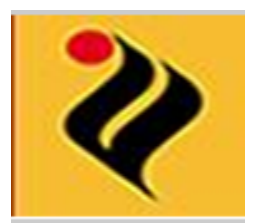

Fig.8. Extracted watermark

Fig 8 shows the extracted watermark from the watermarked image. Here we compared the quality of watermarked image and extracted watermark by the above definition quality assessment metrics such as PSNR, CC and MSSIM. Table 1 shows the values of quality assessment metrics by comparing the quality of watermarked image with original cover image in the invisibility test. Table shows the similarity of each color plane with the original one. The values of PSNR, CC and MSSIM demonstrate the quality of watermarked image is similar to original image. In Table 1 effectiveness test gives the quality of extracting watermark by comparing the original watermark with extracted watermark. The value of $\mathrm{NC}$ is 1 for each color plane demonstrates the quality of extracted watermark is similar to original embedded watermark.

To show that the algorithm gives the good robustness, the different image processing operations are being applied on the watermarked image. The quality of the

extracted watermark from the distorted watermarked image has been analyzed by the quality assessment metrics. Fig. 9 to Fig. 14 show the visual quality of extracting watermark from the watermarked image after Gaussian noise, Pepper salt noise, $90^{\circ}$ rotations, un-sharp filters, projective shearing, sparsity $(128 * 256)$ operation respectively. In Table 2 shows the values of quality assessment metrics after extracted the watermark from the Gaussian Noised, Pepper Salt Noised and rotated watermarked image. Table 2 exhibits that the embedded watermark is robust against the noise and rotation. Table 3 shows the performance of proposed watermark after the filtered, projected and sparse image processing attacks. Values of CC in every test are above 0.8 and that show the extracted watermark is very similarly to original watermark. Values of MSSIM are also above 0.65 in each test that contrast, luminance and the structure of watermarked image are very similar to original one. Values of CC and MSSIM confirm the similarity of extracting watermark with original watermark as well as quality of extracting and watermarked image is very high. In Table 1, Table 2 and Table 3 verify the technique gives good invisibility, effectiveness and robustness.

Table 1. Performance evaluation of proposed method

\begin{tabular}{|c|c|c|}
\hline Test & Invisibility Test & Extraction \\
\hline PSNR & 44.974 & 38.5623 \\
\hline Red_CC & 0.9996 & 1 \\
\hline Gr_CC & 0.9996 & 1 \\
\hline Blue_CC & 0.9991 & 1 \\
\hline Red_MSSIM & 0.9941 & 0.9761 \\
\hline Gr_MSSIM & 0.995 & 0.9981 \\
\hline Blue_MSSIM & 0.9943 & 0.9953 \\
\hline
\end{tabular}

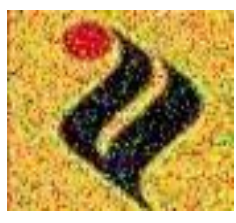

Fig.9. Extracted watermark from noised image (Gaussian) watermarked image

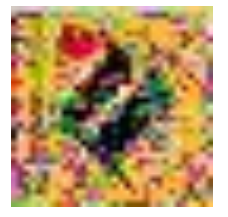

Fig.11. Extracted watermark from $90^{\circ}$ rotated image

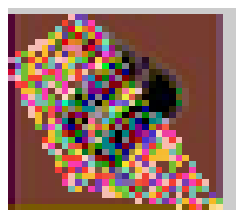

Fig.13. Extracted watermark from projective shearing image

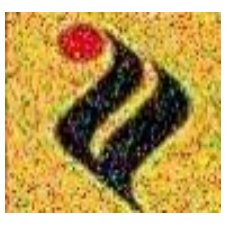

Fig.10. Extracted watermark from noised image (salt)

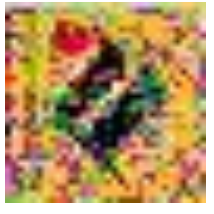

Fig.12.Extracted watermark from filtered image

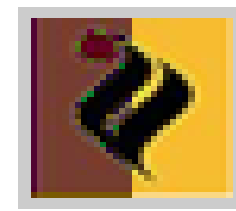

Fig.14. Extracted watermark sparse $(128 * 256)$ image 
Table 2. Performance evaluation against noise addition and rotation

\begin{tabular}{|c|c|c|c|}
\hline Attacks & $\begin{array}{c}\text { Gaussian } \\
\text { Noise }\end{array}$ & $\begin{array}{c}\text { Pepper } \\
\text { Salt Noise }\end{array}$ & $\begin{array}{c}90 \text { Degree } \\
\text { Rotation }\end{array}$ \\
\hline PSNR & 19.631 & 18.953 & 14.649 \\
\hline Red_CC & 0.9596 & 0.9496 & 0.9293 \\
\hline Gr_CC & 0.8979 & 0.8579 & 0.7614 \\
\hline Blue_CC & 0.7136 & 0.7086 & 0.6682 \\
\hline Red_MSSIM & 0.8998 & 0.8783 & 0.8629 \\
\hline Gr_MSSIM & 0.7916 & 0.7716 & 0.7311 \\
\hline Blue_MSSIM & 0.6555 & 0.5955 & 0.6216 \\
\hline
\end{tabular}

Table 3. Performance evaluation against image processing attacks

\begin{tabular}{|c|c|c|c|c|}
\hline Attacks & $\begin{array}{c}\text { Un-sharp } \\
\text { Filtering }\end{array}$ & $\begin{array}{c}\text { Cropping } \\
128^{*} 128\end{array}$ & $\begin{array}{c}\text { Projective } \\
\text { shearing }\end{array}$ & $\begin{array}{c}\text { Sparsity } \\
\left(128^{*} 256\right)\end{array}$ \\
\hline PSNR & 11.232 & 13.089 & 8.5845 & 11.649 \\
\hline Red_CC & 0.8787 & 0.7843 & 0.8555 & 0.8379 \\
\hline Gr_CC & 0.7583 & 0.6339 & 0.7001 & 0.7487 \\
\hline Blue_CC & 0.7025 & 0.5994 & 0.6879 & 0.8122 \\
\hline Red_MSSIM & 0.8073 & 0.7723 & 0.7858 & 0.7912 \\
\hline Gr_MSSIM & 0.6889 & 0.6047 & 0.653 & 0.6939 \\
\hline Blue_MSSIM & 0.6665 & 0.5418 & 0.6338 & 0.7979 \\
\hline
\end{tabular}

To emphasize and to evaluate the performance of the proposed technique the performance of watermark is evaluated on different image processing attacks with various density and variance. Fig. 15 shows the NC and MSSIM values of extracted watermark form the of watermarked image which carries Gaussian noise with different variance.

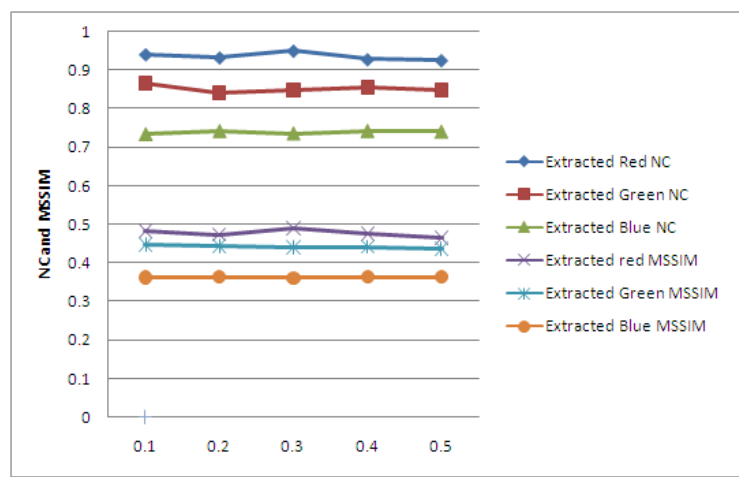

Fig.15. NC and MSSIM of extracted watermark with different Gaussian noise variance

Fig. 16 shows the performance of each color planes with watermark extracted from pepper \& salt noised watermarked image with different density. Form both the Figures is concluded that the most suffer area is blue plane but the human eyes are not sensitive with blue plane. The proposed algorithms gives the robustness from the noise it is verified.

Fig. 17 and Fig 18 show the performance of proposed algorithm rotation and sparse operations. It is also observed form the figures that the values of MSSIM on all plane are very good expect the blue plane. But the visual quality of extracted watermark is visibly good, so it is investigated that the proposed algorithm provides a technique that is robust against the image processing attacks.

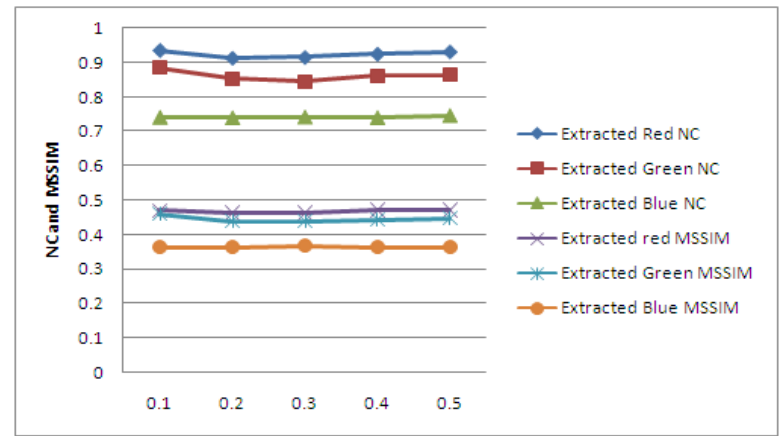

Fig.16. NC and MSSIM of extracted watermark from pepper \& salt noised image with different density

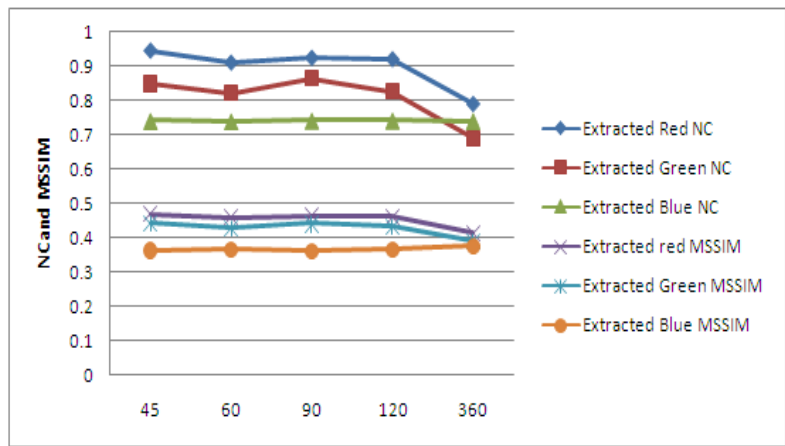

Fig.17. NC and MSSIM of extracted watermark with rotated image with different angle

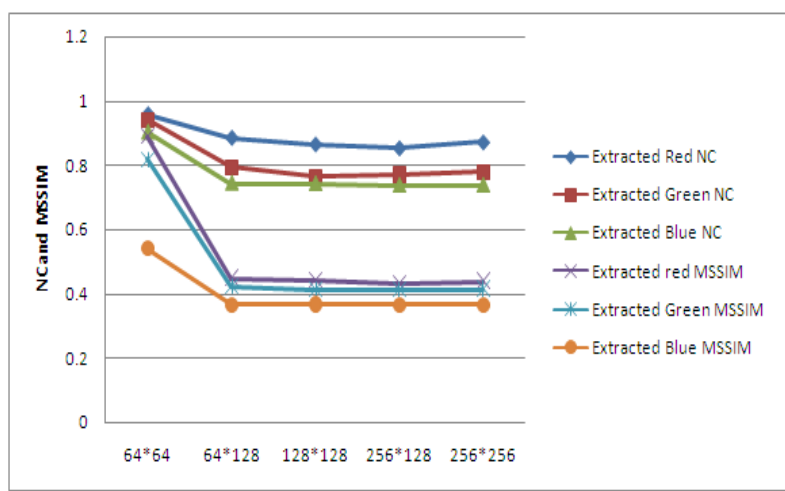

Fig.18. NC and MSSIM of extracted watermark with sparse image with different number of zeros

\section{CONCLUSION}

The semi blind color watermarking technique for embedding the color watermark in RGB image has been presented. The extraction does not need the original cover image at the time of extraction. The bit plane and HVS concepts are used for embedding the watermark into the images. To make the algorithm more secure a cryptographic method has been used by the algorithm. The most significant bit planes of each color plane of watermark image are embedded into the curvelet coefficients of the blue color plane of theoriginal cover image. To test the scheme, the algorithm has been tested against invisibility, effectiveness and robustness. The quality of watermarked image and extracted watermark are being analyzed by the quality assessment metrics such 
as PSNR, CC and MSSIM.

\section{REFERENCES}

[1] Lee, Sin-Joo, and Sung-Hwan Jung. "A survey of watermarking techniques applied to multimedia." Industrial Electronics, 2001. Proceedings. ISIE 2001. IEEE International Symposium on. Vol. 1. IEEE, 2001.

[2] Cox, Ingemar, et al. Digital watermarking and steganography. Morgan Kaufmann, 2007.

[3] Chang, Chin-Chen, Tung-Shou Chen, and Lou-Zo Chung. "A steganographic method based upon JPEG and quantization table modification." Information Sciences 141.1 (2002): 123-138.

[4] Chang, Chin-Chen, Tung-Shou Chen, and Lou-Zo Chung. "A steganographic method based upon JPEG and quantization table modification." Information Sciences 141.1 (2002): 123-138.

[5] Huang, Jiwu, Yun Q. Shi, and Yi Shi. "Embedding image watermarks in DC components." IEEE Transactions on Circuits and Systems for Video Technology 10.6 (2000): 974-979.

[6] C. S LU, S. K. Huang, C. J. Sze, H. Y. M. Liao.: Cocktail watermarking for digital image protection. IEEE Trans. Multimedia. 2000, 2, pp 209-224.

[7] S. H. Wang, Y. P. Lin.: Wavelet tree quantization for copyright protection watermarking, IEEE Trans. Image Process. 2004, 13, pp 154-165.

[8] Gunjal, Baisa L., and Suresh N. Mali. "Strongly robust and highly secured DWT-SVD based color image watermarking: embedding data in all $\mathrm{Y}, \mathrm{U}, \mathrm{V}$ color spaces." International Journal of Information Technology and Computer Science (IJITCS) 4.3 (2012): 1.

[9] P. Tao, A. M. Eskicioglu.: A Robust Multiple Watermarking Scheme in the Discrete Wavelet Transform Domain. Optics East Internet Multimedia Management Systems Conference, Philadelphia, PA, 2004

[10] Gunjal, Baisa L. "Robust, Secure and High Capacity Watermarking Technique based on Image PartitioningMerging Scheme." International Journal of Information Technology and Computer Science (IJITCS) 8.4 (2016): 74.

[11] Do, Minh N., and Martin Vetterli. "The finite ridgelet transform for image representation." IEEE Transactions on image Processing 12.1 (2003): 16-28.

[12] Candes, Emmanuel J., and David L. Donoho. Curvelets: A surprisingly effective nonadaptive representation for objects with edges. Stanford Univ Ca Dept of Statistics, 2000.

[13] Donoho, David L., and Mark R. Duncan. "Digital curvelet transform: strategy, implementation, and experiments." AeroSense 2000. International Society for Optics and Photonics, 2000.

[14] Hien, Thai Duy, et al. "Curvelet-domain image watermarking based on edge-embedding." International Conference on Knowledge-Based and Intelligent Information and Engineering Systems. Springer Berlin Heidelberg, 2007.

[15] Shi, Jianping, and ZhengjunZhai. "Curvelet transform for image authentication." International Conference on Rough Sets and Knowledge Technology. Springer Berlin Heidelberg, 2006.

[16] Lin, C-Y., et al. "Rotation, scale, and translation resilient watermarking for images." IEEE transactions on image processing 10.5 (2001): 767-782.

[17] Solachidis, Vassilios, and Loannis Pitas. "Circularly symmetric watermark embedding in 2-D DFT domain."
IEEE transactions on image processing 10.11 (2001): 1741-1753.

[18] Stankovic, Srdjan, Igor Djurovic, and Ioannis Pitas. "Watermarking in the space/spatial-frequency domain using two-dimensional Radon-Wigner distribution." IEEE Transactions on Image Processing 10.4 (2001): 650-658.

[19] Candès, Emmanuel J., and David L. Donoho. "New tight frames of curvelets and optimal representations of objects with piecewise $\mathrm{C} 2$ singularities." Communications on pure and applied mathematics 57.2 (2004): 219-266.

[20] Donoho, D. L., and E. Candes. "Continuous curvelet transform. II. Discretization and frames." Appl. Comput. Harmon. Anal 19.2 (2005): 198-222.

[21] Hien, Thai Duy, et al. "Curvelet transform based logo watermarking." Innovative Algorithms and Techniques in Automation, Industrial Electronics and Telecommunications. Springer Netherlands, 2007. 305309.

[22] Hien, Thai Duy, et al. "Curvelet-domain image watermarking based on edge-embedding." International Conference on Knowledge-Based and Intelligent Information and Engineering Systems. Springer Berlin Heidelberg, 2007.

[23] Marini, Enrico, et al. "Evaluation of standard watermarking techniques." Electronic Imaging 2007. International Society for Optics and Photonics, 2007.

[24] Kaur, Rupinder. "A medical image watermarking technique for embedding EPR and Its quality assessment using no-reference metrics." International Journal of Information Technology and Computer Science (IJITCS) 5.2 (2013): 73 .

[25] Wang, Zhou, and Alan C. Bovik. "Mean squared error: love it or leave it? A new look at signal fidelity measures." IEEE signal processing magazine 26.1 (2009): 98-117.

[26] Wang, Zhou, et al. "Image quality assessment: from error visibility to structural similarity." IEEE transactions on image processing 13.4 (2004): 600-612.

\section{Authors' Profiles}

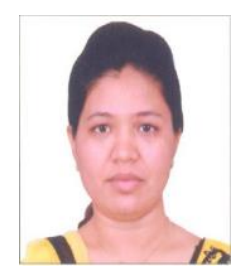

Ranjeeta pursuing Ph.D in Computer Science and Engineering department from Thapar University, Patiala. and working as Associate professor in Chandigarh Engineering College, Landran, Mohali, Punjab. She is having 12 years of teaching experience. She is having 13 national, international and conferences publications. Her area of research is Watermarking in curvelet domain.

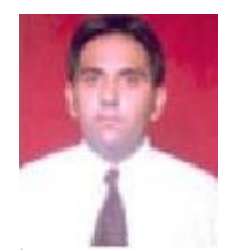

Dr. Sanjay Sharma is currently working as Professor and Head of the department in Electronics and Communication Engineering of Thapar University, India. He has done his B. Tech in ECE from REC in 1993, Jalandhar, M. E. in ECE from TTTI, Chandigarh in 2001 and Ph.D. from PTU, Jalandhar in 2006. He has completed all his education with honors. He has published many papers in various journals and conferences of international repute. He has to his credit the implementation of research projects worth 12000 USD. His main interests are VLSI Signal Processing, Wireless System Design using Reconfigurable Hardware, Digital communication, 


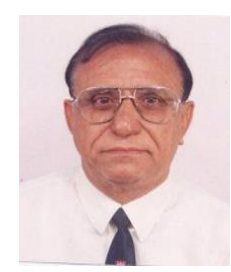

Dr. Lajpat R. Raheja Completed his Ph.D. in Applied Mathematics, from IIT Kharagpur, West Bengal. He has more than 30 years of teaching and research experience. He worked in number of research projects in field of computer sciences, mechanical and naval engineering. $\mathrm{He}$ is recipient of 3 internal awards Alexander von Humboldt Fellowship, West Germany, March1971-June1972, Research Scientist, Institutfür Schiffbau, University of Hamburg, Hamburg, West Germany, Jan.1985-Jan. 1987 and Exchange Program, Department of Naval Architecture, University of Zagreb, Yugoslavia, April-June, 1979.

How to cite this paper: Ranjeeta, Sanjay Sharma, L. R. Raheja,"A Secure and Semi-Blind Technique of Embedding Color Watermark in RGB Image Using Curvelet Domain", International Journal of Information Technology and Computer Science (IJITCS), Vol.9, No.3, pp.62-70, 2017. DOI: 10.5815/ijitcs.2017.03.07 